\section{Global Magnetic Attitude Control of Spacecraft in the Presence of Gravity Gradient}

\author{
MARCO LOVERA \\ Politecnico di Milano \\ Italy
}

ALESSANDRO ASTOLFI, Senior Member, IEEE Imperial College

England

The problem of Earth-pointing attitude control for a spacecraft with magnetic actuators is addressed and a novel approach to the problem is proposed, which guarantees almost global closed loop stability of the desired relative attitude equilibrium for the spacecraft. Precisely, a proportional derivative (PD)-like state feedback control law is employed together with a suitable adaptation mechanism for the controller gain. Simulation results are presented, which illustrate the performance of the proposed control law.

Manuscript received May 21, 2004; revised May 2 and September 6, 2005; released for publication February 1, 2006.

IEEE Log No. T-AES/42/3/884428.

Refereeing of this contribution was handled by M. Ruggieri.

This work was supported by the ASI project, "Global Attitude Determination and Control using Magnetic Sensor and Actuators."

Authors' addresses: M. Lovera, Dipartimento di Elettronica e Informazione, Politecnico di Milano, Piazza Leonardo da Vinci 32, 20133 Milano, Italy, E-mail: (lovera@elet.polimi.it); A. Astolfi, Dept. of Electrical and Electronic Engineering, Imperial College, Exhibition Road, SW7 2BT London, England.

$\overline{0018-9251 / 06 / \$ 17.00 ~(c) ~} 2006$ IEEE

\section{INTRODUCTION}

Magnetic coils have been extensively used since the early 1960s as a simple and reliable technology to implement attitude control actuators in low Earth orbit satellites (see, e.g., [1] or [2] and the references therein). Such actuators operate on the basis of the interaction between the geomagnetic field and a set of three orthogonal current-driven coils; this has a number of implications which make the magnetic spacecraft control problem significantly different from the conventional attitude regulation one. The main difficulty is due to the fact that it is not possible (by means of magnetic actuators) to provide three independent control torques at each time instant. In addition, the behaviour of these actuators is intrinsically time varying, as the control mechanism relies on the variations of the Earth magnetic field along the spacecraft orbit.

A considerable amount of work has been dedicated in recent years to the problems of analysis and design of magnetic control laws in the linear case, i.e., control laws for nominal operation of a satellite near its equilibrium attitude, using either periodic optimal control (see, e.g., [3], [4], [5], [6]) or other techniques aiming at developing suitable time-varying controllers $[7,8]$.

However, the global formulation of the problem is also of considerable interest, but has not been studied to a comparable extent. In particular, in [9], [10], and [11] the attitude regulation problem for Earth-pointing spacecraft has been addressed exploiting the (quasi) periodic behaviour of the system, hence resorting to standard passivity arguments to prove local asymptotic stabilisability of open loop equilibria. In [12] similar arguments have been used to analyse a state feedback control law for the particular case of an inertially spherical spacecraft. More recently, in [13], [14], and [15] the case of inertial pointing has been considered, and a solution to the global stabilization problem by means of full (or partial) state feedback has been studied.

The aim of this paper is to show how stability conditions similar to those given in [13] and [14] can be derived for control laws achieving Earth pointing for magnetically actuated spacecraft, taking also into account the effect of gravity gradient torques. For this problem, an almost global stabilisation result is given for the case of full state feedback, resorting to an adaptive control approach. Moreover, the results presented herein do not rely on the (frequently adopted) periodicity assumption for the geomagnetic field along the considered orbit, which is correct only to first approximation (see, e.g., [16]).

The paper is organised as follows. In Section II the equations for the attitude motion for a magnetically actuated spacecraft are presented. Section III is dedicated to the state feedback attitude control 
problem, while some simulation results are presented in Section IV.

\section{SPACECRAFT MODEL}

\section{A. Coordinate Frames}

For the purpose of the present analysis, the following reference systems are adopted.

1) Earth-centered inertial reference axes (ECI). The origin of these axes is in the Earth's centre. The $\mathrm{X}$-axis is parallel to the line of nodes. The Z-axis is parallel to the Earth's geographic north-south axis and pointing north. The Y-axis completes the right-handed orthogonal triad.

2) Orbital axes $\left(X_{0}, Y_{0}, Z_{0}\right)$. The origin of these axes is in the satellite centre of mass. The $\mathrm{X}$-axis points to the Earth's centre; the Y-axis points in the direction of the orbital velocity vector. The Z-axis is normal to the satellite orbit plane.

3) Satellite body axes. The origin of these axes is in the satellite centre of mass; the axes are assumed to coincide with the body's principal inertia axes.

In this paper only the case of a spacecraft in a circular orbit is considered; the (constant) orbital angular rate will be denoted by $\omega_{0}$. Finally, in the following the unit vectors corresponding to the orbital axes will be denoted with $e_{x}, e_{y}$, and $e_{z}$, respectively, with the superscript ${ }^{o}\left({ }^{b}\right)$ when considering the components of the unit vectors along the orbital (body) axes.

\section{B. Dynamics}

The attitude dynamics of a spacecraft subject to gravity gradient can be expressed (in the body frame) as [16]

$$
I \dot{\omega}=S(\omega) I \omega+3 \omega_{0}^{2} S\left(I e_{x}^{b}\right) e_{x}^{b}+T_{\text {coils }}+T_{\text {dist }}
$$

where $\omega \in \mathbb{R}^{3}$ is the vector of spacecraft angular rates, $I=\operatorname{diag}\left[I_{x}, I_{y}, I_{z}\right] \in \mathbb{R}^{3 \times 3}$ is the inertia matrix, $S(\omega)$ is given by

$$
S(\omega)=\left[\begin{array}{ccc}
0 & \omega_{z} & -\omega_{y} \\
-\omega_{z} & 0 & \omega_{x} \\
\omega_{y} & -\omega_{x} & 0
\end{array}\right]
$$

$T_{\text {coils }} \in \mathbb{R}^{3}$ is the vector of external torques induced by the magnetic coils and $T_{\text {dist }} \in \mathbb{R}^{3}$ is the vector of external disturbance torques.

\section{Relative Kinematics}

We are concerned here with the dynamics of an Earth-pointing satellite, so the focus will be on the relative kinematics rather than on the inertial kinematics. In other words, we are concerned with representations of the attitude of the spacecraft with respect to the (rotating) orbital axes.

The attitude kinematics is described in terms of the four Euler parameters (or quaternions, see, e.g., [16]), which lead to the following representation for the relative attitude kinematics

$$
\dot{q}=\tilde{W}(q) \omega_{r}
$$

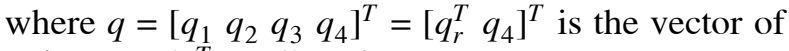
unit norm $\left(q^{T} q=1\right)$ Euler parameters,

$$
\tilde{W}(q)=\frac{1}{2}\left[\begin{array}{ccc}
q_{4} & -q_{3} & q_{2} \\
q_{3} & q_{4} & -q_{1} \\
-q_{2} & q_{1} & q_{4} \\
-q_{1} & -q_{2} & -q_{3}
\end{array}\right]
$$

and $\omega_{r}=\omega-\omega_{t}=\omega+\omega_{0} e_{z}^{b}$ is the satellite angular rate relative to the orbital axes, in body frame. Letting $A(q)$ be the attitude matrix relating the orbital and the body frames, one has that

$$
e_{x}^{b}=A(q) e_{x}^{o}=A(q)\left[\begin{array}{l}
1 \\
0 \\
0
\end{array}\right]
$$

and similarly for $e_{y}^{b}, e_{z}^{b}$. Finally, note that $A(q)=\mathcal{I}_{3}$ (where $\mathcal{I}_{3}$ is the identity matrix of dimension 3 ) for $q= \pm \bar{q}= \pm\left[\begin{array}{llll}0 & 0 & 0 & 1\end{array}\right]^{T}$.

\section{Magnetic Coils}

The magnetic attitude control torques are generated by a set of three magnetic coils, aligned with the spacecraft principal inertia axes, which generate torques according to the law

$$
T_{\text {coils }}=m_{\text {coils }} \times \tilde{b}(t)=S(\tilde{b}(t)) m_{\text {coils }}
$$

where $\times$ denotes the vector cross product, $m_{\text {coils }} \in$ $\mathbb{R}^{3}$ is the vector of magnetic dipoles for the three coils, and $\tilde{b}(t) \in \mathbb{R}^{3}$ is the vector formed with the components of the Earth's magnetic field in the body frame of reference. Note that the vector $\tilde{b}(t)$ can be expressed in terms of the attitude matrix $A(q)$ (see [16] for details) and of the magnetic field vector expressed in the inertial coordinates, namely $\tilde{b}_{0}(t)$, as

$$
\tilde{b}(t)=A(q) \tilde{b}_{0}(t)
$$

and that the orthogonality of $A(q)$ implies $\|\tilde{b}(t)\|=$ $\left\|\tilde{b}_{0}(t)\right\|$. Since $S(\tilde{b}(t))$ is structurally singular, as mentioned in the introduction, magnetic actuators do not provide full controllability of the system at each time instant. In particular, it is easy to see that $\operatorname{rank}(S(\tilde{b}(t)))=2\left(\right.$ since $\left\|\tilde{b}_{0}(t)\right\| \neq 0$ along all orbits of practical interest for magnetic control) and that the kernel of $S(\tilde{b}(t))$ is given by the vector $\tilde{b}(t)$ itself, 
i.e., at each time instant it is not possible to apply a control torque along the direction of $\tilde{b}(t)$.

If a preliminary feedback of the form

$$
m_{\text {coils }}=\frac{1}{\left\|\tilde{b}_{0}(t)\right\|^{2}} S^{T}(\tilde{b}(t)) v
$$

is applied to the system, where $u \in \mathbb{R}^{3}$ is a new control vector, the overall dynamics can be written as

$$
\begin{aligned}
\dot{q} & =W(q) \omega_{b} \\
J_{0} \dot{\omega}_{b} & =S(\omega) J_{0} \omega+\Gamma(t) v
\end{aligned}
$$

where $\Gamma(t)=S(b(t)) S^{T}(b(t)) \geq 0$ and $b(t)=$ $\left(1 /\left\|\tilde{b}_{0}(t)\right\|\right) \tilde{b}(t)=(1 /\|\tilde{b}(t)\|) \tilde{b}(t)$. Similarly, let $\Gamma_{0}(t)=$ $S\left(b_{0}(t)\right) S^{T}\left(b_{0}(t)\right) \geq 0$ and $b_{0}(t)=\left(1 /\left\|\tilde{b}_{0}(t)\right\|\right) \tilde{b}_{0}(t)$. Note, also, that $\Gamma(t)$ can be written as $\Gamma(t)=\mathcal{I}_{3}-b(t) b(t)^{T}$, where $\mathcal{I}_{3}$ is the $3 \times 3$ identity matrix. We now prove a preliminary result which will be exploited in the next section.

LEMMA 1 Consider the system (9) and assume that the considered orbit for the spacecraft satisfies the condition

$$
\bar{\Gamma}_{0}=\lim _{T \geq \infty} \frac{1}{T} \int_{0}^{T} S\left(b_{0}(t)\right) S^{T}\left(b_{0}(t)\right) d t>0 .
$$

Then, there exists $\omega_{M}>0$ such that if $\left\|\omega_{r}\right\|<\omega_{M}$ for all $t>\bar{t}$, for some $0<\bar{t}<\infty$, then

$$
\bar{\Gamma}=\lim _{T \geq \infty} \frac{1}{T} \int_{0}^{T} S(b(t)) S^{T}(b(t)) d t>0
$$

along the trajectories of the system (9).

PROOF Consider first the particular case $\omega_{r}=0$, which implies that $q=\bar{q}=$ const. If $\bar{\Gamma}$ is singular there exists a non-zero vector $\bar{v}$ such that

$$
\bar{v}^{T} \bar{\Gamma} \bar{v}=0
$$

and $v_{0}=A(\bar{q})^{T} \bar{v}$. However, (11) and (7) imply that

$$
v_{0}^{T} \bar{\Gamma}_{0} v_{0}=0
$$

which contradicts the assumption. Finally, continuity arguments suffice to guarantee that (10) holds provided that $\omega$ is sufficiently small for all $t>\bar{t}$, for some $0<\bar{t}<\infty$.

Lemma 1 lends itself to a simple physical interpretation. Condition $\operatorname{det}(\bar{\Gamma})=0$ defines the set of all trajectories along which average controllability is lost. Since, under the assumptions of Lemma 1 , we have that $\bar{\Gamma}_{0}>0$, the fact that $\operatorname{det}(\bar{\Gamma})=0$ implies that the attitude trajectory of the satellite, combined with the natural on-orbit variability of $b_{0}(t)$, gives rise to a situation in which the average gain of the system is singular. The interpretation of the Lemma is the following. Since the natural variability of $b_{0}(t)$ is characterised by a dominant frequency $\omega_{0}$, the condition $\operatorname{det}(\bar{\Gamma})=0$ can only arise whenever the angular rate of the spacecraft is sufficiently large: indeed if the modulus of angular rate is smaller than $\omega_{0}$, then the attitude motion of the satellite cannot "compensate" for the natural variability of $b_{0}(t)$, hence average controllability in the sense of (10) is guaranteed for sufficiently small $\omega$.

REMARK 1 Some further insight in the condition $\bar{\Gamma}_{0}>0$ can be gained by resorting to a simplified geomagnetic field model in order to derive an analytical expression for $\bar{\Gamma}_{0}$. For the sake of simplicity, and given that we are only concerned with the rank of the average gain $\bar{\Gamma}_{0}$, the calculation will be carried out using the unnormalised magnetic field vector $\tilde{b}_{0}$. To this purpose, note that (see [17]) a dipole approximation of the Earth's magnetic field, together with the assumptions of no Earth rotation and no orbit precession, yields the following periodic model for the magnetic field vector, as expressed in orbit coordinates:

$$
\tilde{b}_{0}=\frac{\mu_{f}}{a^{3}}\left[\begin{array}{c}
2 \sin \left(\omega_{0} t\right) \sin \left(i_{m}\right) \\
\cos \left(\omega_{0} t\right) \sin \left(i_{m}\right) \\
\cos \left(i_{m}\right)
\end{array}\right]
$$

where $\mu_{f}=7.910^{15} \mathrm{~Wb} \mathrm{~m}$ is the dipole strength, $a$ is the orbit semimajor axis, and $i_{m}$ is the orbit's inclination with respect to the geomagnetic equator. Using such a simplified model for the geomagnetic field leads to the closed form expression for the average gain

$$
\begin{aligned}
& \lim _{T \geq \infty} \frac{1}{T} \int_{0}^{T} S\left(\tilde{b}_{0}(t)\right) S^{T}\left(\tilde{b}_{0}(t)\right) d t= \\
& \frac{\mu_{f}}{a^{3}}\left[\begin{array}{ccc}
\cos \left(i_{m}\right)^{2}+\frac{1}{2} \sin \left(i_{m}\right)^{2} & 0 & 0 \\
0 & \cos \left(i_{m}\right)^{2}+2 \sin \left(i_{m}\right)^{2} & 0 \\
0 & 0 & \frac{5}{2} \sin \left(i_{m}\right)^{2}
\end{array}\right] .
\end{aligned}
$$

Clearly, the condition number of this matrix (i.e., the ratio between the largest and the smallest singular value of the matrix) is a function of the orbit inclination. A plot of the inverse of the condition number of the matrix is shown in Fig. 1, from which it can be seen that, as expected, controllability issues can arise only for low inclination orbits, i.e., when the eigenvalue given by $(5 / 2) \sin \left(i_{m}\right)^{2}$ becomes close to zero.

\section{STATE FEEDBACK STABILIZATION}

In this section an almost globally convergent adaptive PD-like control law for Earth-pointing magnetic attitude regulation is proposed. In order to prove global convergence for the control scheme that is presented, some background on the so-called averaging theory must be introduced. The interested reader is referred to [18] for a more detailed treatment of this topic. 


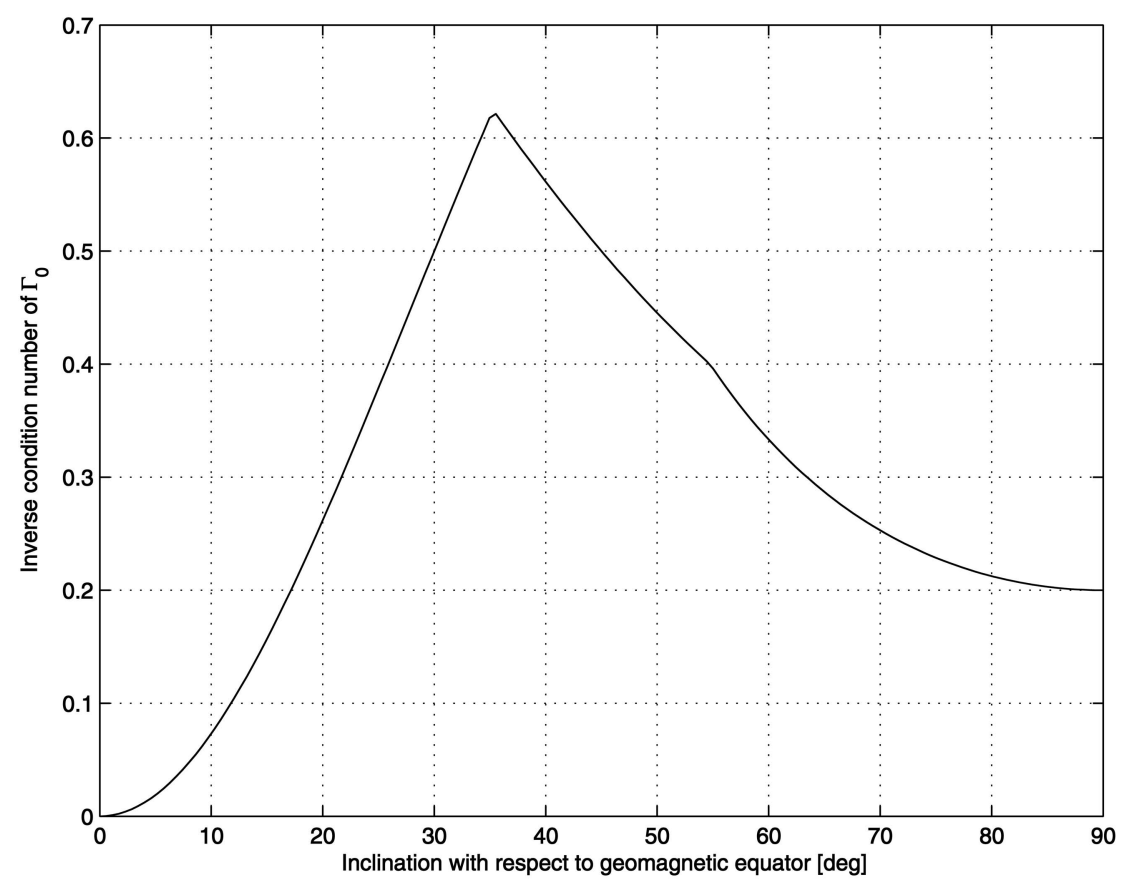

Fig. 1. Inverse of condition number of $\bar{\Gamma}_{0}$ as a function of orbit inclination $i_{m}$.

DEFINITION 1 A continuous, bounded function $g(t, x)$ is said to have an average $g_{\text {av }}(x)$ if the limit

$$
g_{\mathrm{av}}(x)=\lim _{T \rightarrow \infty} \frac{1}{T} \int_{t}^{t+T} g(x, \tau) d \tau
$$

exists and

$$
\left\|\frac{1}{T} \int_{t}^{t+T} g(x, \tau) d \tau-g_{\mathrm{av}}(x)\right\| \leq k \sigma(T), \quad \forall \quad t, x
$$

where $k$ is a positive constant and $\sigma(T)$ is a strictly decreasing, continuous, bounded function such that $\sigma(T) \rightarrow 0$ as $T \rightarrow \infty$.

Consider now the system

$$
\dot{x}=\varepsilon f(t, x, \varepsilon)
$$

$\varepsilon>0$, and suppose that $f(t, x, 0)$ has the average $f_{\mathrm{av}}$.

Then, under suitable assumptions (see [18] for details) it is possible to show that if $f(t, 0, \varepsilon)=0$ and the origin of the averaged system

$$
\dot{x}=\varepsilon f_{\mathrm{av}}(x, \varepsilon)
$$

is exponentially stable, then there exists a positive constant $\varepsilon^{*}$ such that for all $0<\varepsilon<\varepsilon^{*}$ the origin of the original system will be exponentially stable.

In the following, we take advantage of the fact that Lemma 1 shows that for sufficiently small angular rates the system (9) has "average" controllability properties as expressed by the full rank of the matrix $\bar{\Gamma}$. This fact allows for the successful application of averaging theory and plays a major role in the derivation of the following, preliminary result.
PROPOSITION 1 Consider the system (9) and the control law

$$
u=-\varepsilon k_{v} \omega_{r}
$$

Suppose that $0<\bar{\Gamma}_{0}<\mathcal{I}_{3}$. Then, for all $\varepsilon>0$ and $k_{v}>0$ there exists $\bar{t}>0$ such that for all $t>\bar{t}$

$$
\bar{\Gamma}(t)=\frac{1}{t} \int_{0}^{t} \Gamma(\tau) d \tau>0
$$

Proof Consider the function (see also [19], [20])

$$
\begin{aligned}
V_{1}= & \frac{\lambda}{2}\left[\omega_{r}^{T} I \omega_{r}+3 \omega_{0}^{2}\left(e_{x}^{T} I e_{x}-I_{x}\right)+\omega_{0}^{2}\left(I_{z}-e_{z}^{T} I e_{z}\right)\right] \\
& -\frac{1}{2} \omega_{r}^{T} I A(q) M_{0}(t) A(q)^{T} I \omega_{r}
\end{aligned}
$$

where $\lambda>0$,

$$
M_{0}(t)=\int_{0}^{t}\left(b_{0}(\tau) b_{0}(\tau)^{T}-N_{0}\right) d \tau
$$

and $N_{0} \geq 0$ is a constant matrix. The assumption $\bar{\Gamma}_{0}<\mathcal{I}_{3}$ implies that it is possible to select $N_{0}$ such that $-\sigma \mathcal{I}_{3} \leq M_{0}(t) \leq \sigma \mathcal{I}_{3}$ for some positive $\sigma$. Note that $V_{1}$ is positive definite for sufficiently large $\lambda$. The time derivative of $V_{1}$ is given by

$$
\begin{aligned}
\dot{V}_{1}= & -\omega_{r}^{T} A(q) Q A(q)^{T} \omega_{r}-\omega_{r}^{T} I M(t)\left(S\left(I \omega_{t}\right)\right. \\
& \left.+S\left(\omega_{t}\right) I+I S\left(\omega_{t}\right)\right) \omega_{r} \\
& -\omega_{r}^{T} I M(t)\left(S\left(I \omega_{t}\right) I \omega_{t}+T_{g g}\right)
\end{aligned}
$$


where

$$
\begin{aligned}
Q=( & \varepsilon k_{v} \lambda \Gamma_{0}(t)+A(q)^{T} I \\
& \left.\times\left(-\frac{\varepsilon k_{v}}{2} M(t) \Gamma(t)-\frac{\varepsilon k_{v}}{2} \Gamma(t) M(t)+b b^{T}-N\right) I A(q)\right) .
\end{aligned}
$$

Introduce the time-varying vectors $b_{1}(t)$ and $b_{2}(t)$ such that $b_{i}^{T} b_{j}=\delta_{i j}$, where $\delta_{i j}$ is the Kronecker delta and $i, j=0,1,2$, and let

$$
\tilde{Q}=\left[\begin{array}{l}
b_{0}^{T} \\
b_{1}^{T} \\
b_{2}^{T}
\end{array}\right] Q\left[\begin{array}{lll}
b_{0} & b_{1} & b_{2}
\end{array}\right] .
$$

Then, it can be shown that there exists a $\lambda>0$ and sufficiently large such that $\tilde{Q}$ (and, therefore, $Q$ ) is positive definite. This, in turn, implies that for any $\omega_{M}>0$ there exists $\lambda>0$ such that $\left\|\omega_{r}\right\|<\omega_{M}$ for sufficiently large $t$, and therefore, by Lemma 1 , $\bar{\Gamma}>0$.

The main result of this paper is given in the following Proposition.

Proposition 2 Consider the system (9) and the control law

$$
u= \begin{cases}-\varepsilon k_{v} \omega_{r}, & t \leq \bar{t} \\ -\hat{\Gamma}_{\mathrm{av}}^{-1}\left(\varepsilon^{2} k_{p} q_{r}+\varepsilon k_{v} \omega_{r}\right), & t>\bar{t}\end{cases}
$$

where

$$
\dot{\hat{\Gamma}}_{\mathrm{av}}=\frac{1}{t} \Gamma-\frac{1}{t} \hat{\Gamma}_{\mathrm{av}}, \quad t>0
$$

and

$$
\hat{\Gamma}_{\mathrm{av}}(0)=\Gamma(0) .
$$

Then there exist $\varepsilon^{*}>0, k_{p}>0, k_{v}>0$ such that for any $0<\varepsilon<\varepsilon^{*}$ the control law renders the equilibrium $\left(q, \omega_{r}\right)=(\bar{q}, 0)$ of the closed loop system (9)-(26)

locally exponentially stable. Moreover, all trajectories of the closed loop system (9)-(26) converge to the points $\left(q, \omega_{r}\right)=( \pm \bar{q}, 0)$.

PROOF Proposition 1 ensures that the application of the control law (26) for $t \leq \bar{t}$ leads to $\bar{\Gamma}(t)>0$ for all $t>\bar{t}$. Note that the solution of (27) is given by

$$
\hat{\Gamma}_{\mathrm{av}}(t)=\frac{1}{t} \int_{0}^{t} \Gamma(\tau) d \tau
$$

so Proposition 1 also implies that $\lim _{t \rightarrow \infty} \hat{\Gamma}_{\mathrm{av}}(t)=\Gamma_{\mathrm{av}}$. Introduce now the coordinates transformation

$$
z_{1}=q, \quad z_{2}=\frac{\omega}{\varepsilon}
$$

(so that $z_{1 r}=q_{r}, z_{14}=q_{4}, z_{2 r}=\omega_{r} / \varepsilon$ ), in which the closed loop system (9)-(26) for $t>\bar{t}$ is described by the equations

$$
\begin{aligned}
\dot{z}_{1}= & \varepsilon \tilde{W}\left(z_{1}\right) z_{2 r} \\
I \dot{z}_{2}= & \varepsilon S\left(z_{2}\right) I z_{2}+\varepsilon 3 z_{0}^{2} S\left(I e_{x}^{b}\right) e_{x}^{b} \\
& +\varepsilon \Gamma(t) \hat{\Gamma}_{\mathrm{av}}^{-1}(t)\left(-k_{p} z_{1 r}-k_{v} z_{2 r}\right) .
\end{aligned}
$$

System (31) satisfies all the hypotheses for the applicability of the generalised averaging theory (see [18, Theorem 10.5]), which yields the averaged system

$$
\begin{aligned}
\dot{z}_{1}= & \varepsilon \tilde{W}\left(z_{1}\right) z_{2 r} \\
I \dot{z}_{2}= & \varepsilon S\left(z_{2}\right) I z_{2}+3 \varepsilon z_{0}^{2} S\left(I e_{x}^{b}\right) e_{x}^{b} \\
& +\varepsilon \bar{K}\left(-k_{p} z_{1 r}-k_{v} z_{2 r}\right)
\end{aligned}
$$

where $z_{0}=\omega_{0} / \varepsilon$

$$
\bar{K}=\lim _{T \rightarrow \infty} \frac{1}{T} \int_{\bar{t}}^{T} \Gamma(t) \hat{\Gamma}_{\mathrm{av}}^{-1}(t) d t .
$$

We now prove that $\bar{K}=\mathcal{I}_{3}$. For, note that from (27) one has

$$
\hat{\Gamma}_{\mathrm{av}}(t)=\Gamma_{\mathrm{av}}+\Delta(t)
$$

with

$$
\lim _{t \rightarrow \infty}\|\Delta(t)\|=0
$$

and

$$
\hat{\Gamma}_{\mathrm{av}}^{-1}(t)=\Gamma_{\mathrm{av}}^{-1}-E(t)
$$

with

$$
\lim _{t \rightarrow \infty}\|E(t)\|=0
$$

so that $\bar{K}$ can be written as

$$
\bar{K}=\mathcal{I}_{3}+\frac{1}{T} \int_{\bar{t}}^{T} \Gamma(t) E(t) d t
$$

and the boundedness of $E(t)$ ensures that

$$
\lim _{T \rightarrow \infty} \frac{1}{T} \int_{\bar{t}}^{T} \Gamma(t) E(t) d t=0 .
$$

Finally, consider the function

$V_{3}=\frac{1}{2}\left[z_{2 r}^{T} I z_{2 r}+3 z_{0}^{2}\left(e_{x}^{T} I e_{x}-I_{x}\right)+z_{0}^{2}\left(I_{z}-e_{z}^{T} I e_{z}\right)+2 k_{p}\left(1-z_{14}\right)\right]$

and note that for sufficiently large $k_{p}$ function $V_{3}$ is positive definite. The time derivative of $V_{3}$ along the trajectories of the closed loop system (9)-(26) is given by (see [20])

$$
\dot{V}_{3}=z_{2 r}^{T} I \dot{z}_{2 r}+3 z_{0}^{2} e_{x}^{T} I \dot{e}_{x}-z_{0}^{2} e_{z}^{T} I \dot{e}_{z}+k_{p} z_{2 r}^{T} z_{1 r} .
$$

Now, note that from (5) one has that

$$
\dot{e}_{x}^{b}=S\left(\omega_{r}\right) e_{x}^{b}
$$

and

$$
\dot{e}_{z}^{b}=S\left(\omega_{r}\right) e_{z}^{b}
$$




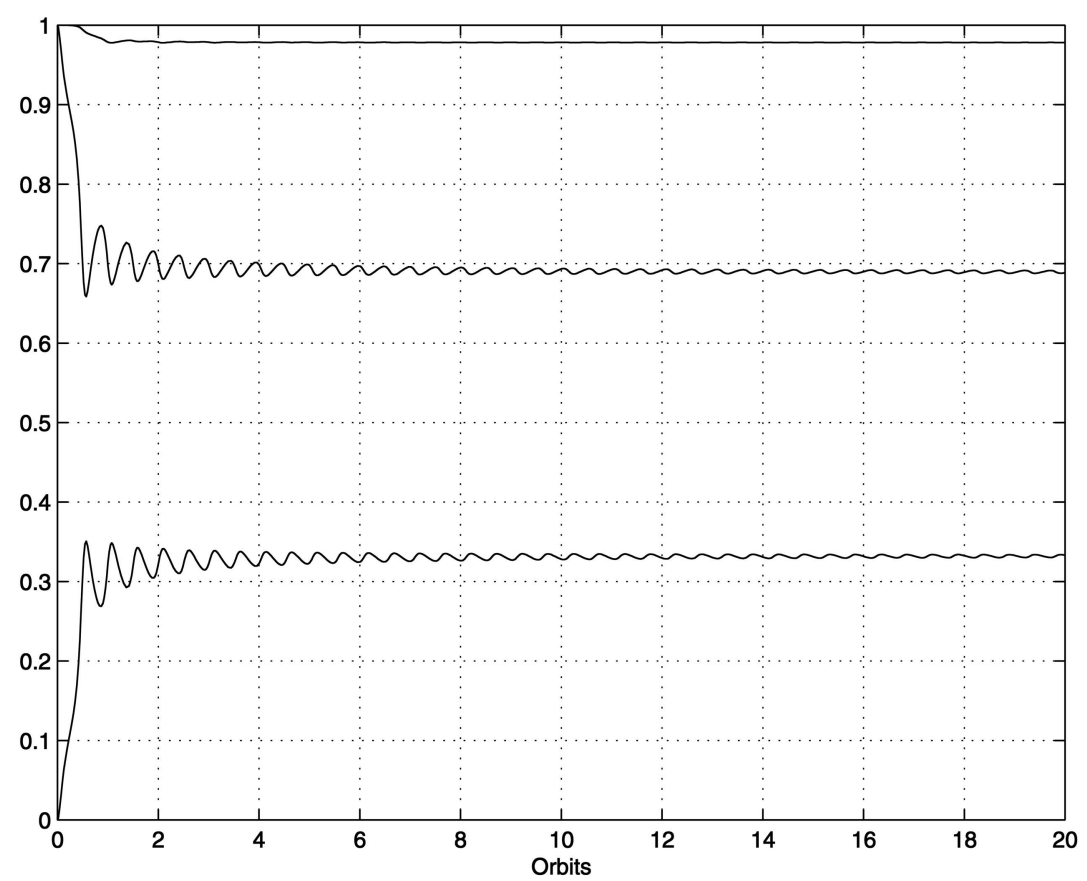

Fig. 2. Eigenvalues of $(1 / T) \int_{0}^{T} \Gamma_{0}(t) d t$ for considered orbit.

Similarly, since $\omega_{r}=\omega+\omega_{0} e_{z}^{b}$, one has

$$
\begin{aligned}
I \dot{z}_{2 r}= & I \dot{z}_{2}+z_{0} I \dot{e}_{z}^{b}=I \dot{z}_{2}+z_{0} S\left(z_{2 r}\right) e_{z}^{b} \\
= & S\left(z_{2 r}\right) I\left(z_{2 r}-z_{0} e_{z}^{b}\right)-z_{0} S\left(e_{z}^{b}\right) I\left(z_{2 r}-z_{0} e_{z}^{b}\right) \\
& +3 z_{0}^{2} S\left(I e_{x}^{b}\right) e_{x}^{b}+u+z_{0} I S\left(z_{2 r}\right) e_{z}^{b} .
\end{aligned}
$$

Therefore, (41) can be equivalently written as

$$
\dot{V}_{3}=z_{2 r}^{T} u-k_{p} z_{2 r}^{T} z_{1 r}=-k_{v} z_{2 r}^{T} z_{2 r} .
$$

As $\dot{V}_{3} \leq 0$, one has that $z_{2 r} \rightarrow 0$ and therefore for sufficiently large $k_{p}$ also $z_{1 r} \rightarrow 0$.

\section{SIMULATION RESULTS}

The considered spacecraft has an inertia matrix given by $I=\operatorname{diag}[5,60,70] \mathrm{kg} \mathrm{m}^{2}$, and operates in a near polar ( $87^{\circ}$ inclination) orbit with an altitude of $450 \mathrm{~km}$ and a corresponding orbit period of about $5600 \mathrm{~s}$. Note that the first element of the inertia matrix is much smaller than the other two: such an inertia matrix is representative of a small satellite with a long gravity gradient boom along the $x$ axis (see, e.g., [21]). It is worth, first of all, to check that the assumption $0<\bar{\Gamma}_{0}<\mathcal{I}_{3}$, which plays a major role in the formulation of the magnetic attitude control problem, is satisfied in practice. In order to illustrate this, in Fig. 2 a time history of the eigenvalues of $(1 / T) \int_{0}^{T} \Gamma_{0}(t) d t$ computed for the considered orbit is presented. As can be seen from the figure, $(1 / T) \int_{0}^{T} \Gamma_{0}(t) d t$ converges to a $\bar{\Gamma}_{0}$ which satisfies the assumption.
For the considered spacecraft two simulations have been carried out: the first one is related to the acquisition of the target attitude $\bar{q}$ from an initial condition characterized by a high initial angular rate; the second one illustrates the behaviour of the proposed control strategy when recovering the desired target attitude from an initial condition corresponding to the initial attitude $\left[\begin{array}{llll}0 & 0 & 1 & 0\end{array}\right]^{T}$ and zero relative angular rate. In both cases, according to Proposition 2 , the satellite is initially subject to a purely derivative control law. In order to take into account the effect of disturbance torques on the behaviour of the controlled spacecraft, a residual magnetic dipole $m_{0}=\left[\begin{array}{lll}1 & 1 & 1\end{array}\right]^{T}$ (chosen according to the guidelines given in [22]) has been considered, together with the effect of gravity gradient torques.

The results of the attitude acquisition simulation are displayed in Figs. 3 and 4, from which the good performance of the control law, with parameters $\varepsilon=0.001, k_{p}=500\left(\mathrm{~A} \mathrm{~m}^{2}\right), k_{v}=200 \mathrm{~A} \mathrm{~m}^{2} /(\mathrm{rad} / \mathrm{s})$, can be seen. In particular, as can be seen from the figures, after an initial transient during which the control law essentially reduces the kinetic energy of the spacecraft (hence the decreasing frequency of the initial oscillations of the quaternion components), the desired Earth-pointing attitude is achieved.

Finally, we consider a simulation in which the controller has to recover the desired attitude from an "upside down" initial attitude, i.e., a situation in which the spacecraft is initially in one of the undesired stable open loop equilibria of relative motion (see [19]). As can be seen from Figs. 5 and 6, the proposed adaptive control law, with parameters $\varepsilon=0.001$, $k_{p}=500\left(\mathrm{~A} \mathrm{~m}^{2}\right), k_{v}=200 \mathrm{~A} \mathrm{~m}^{2} /(\mathrm{rad} / \mathrm{s})$, can bring 

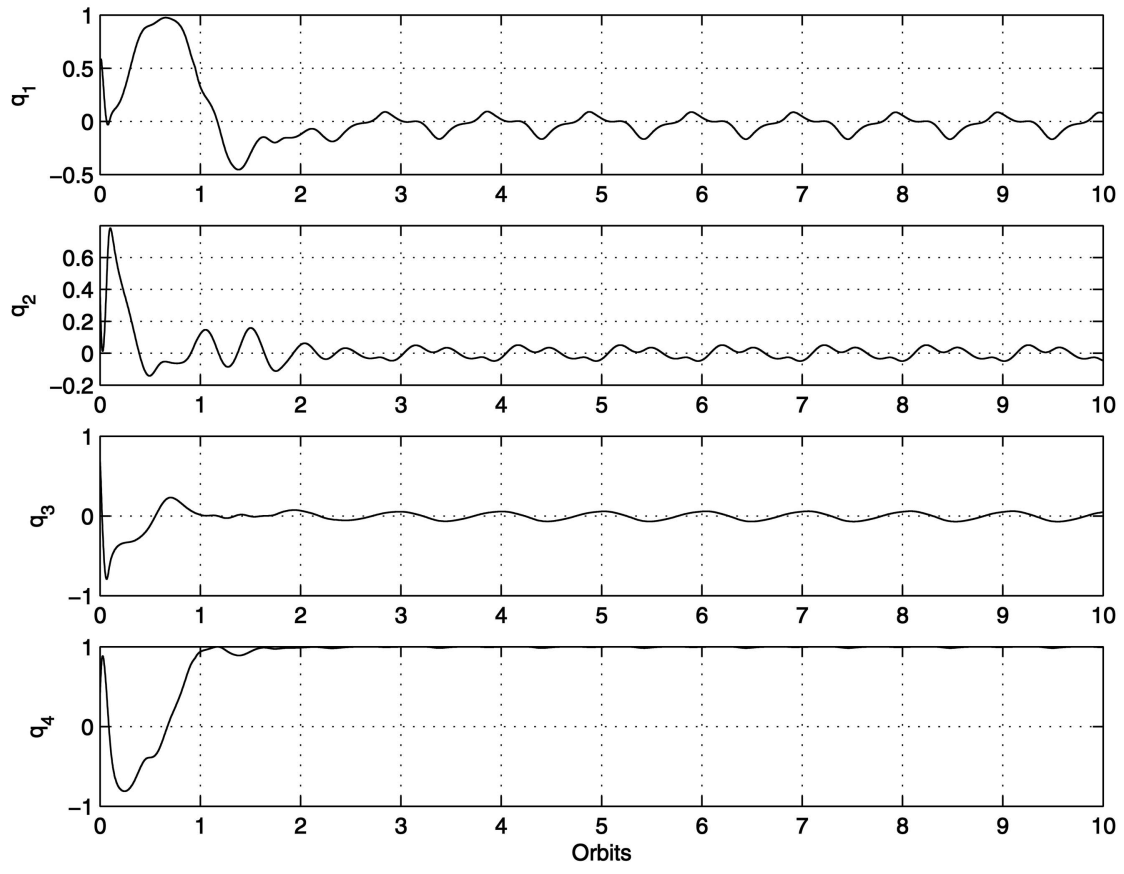

Fig. 3. Time evolution of attitude quaternion during attitude acquisition.
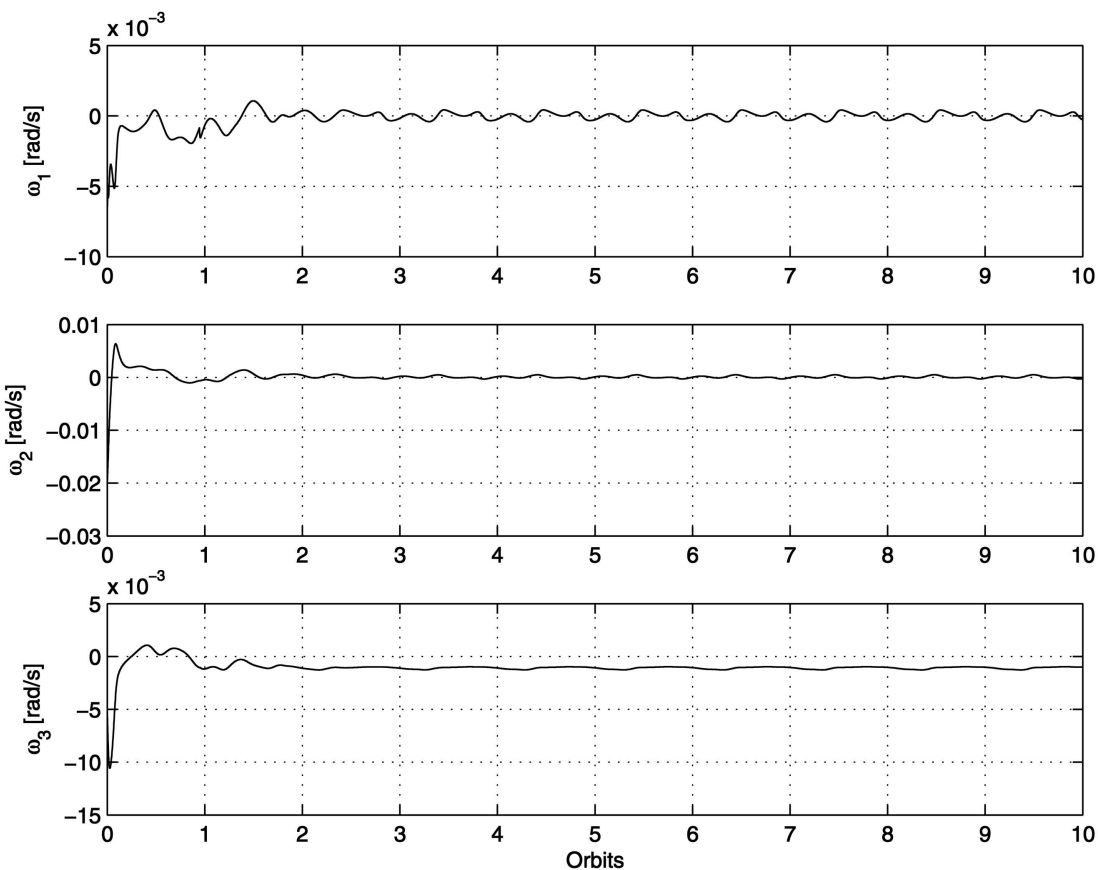

Fig. 4. Time evolution of absolute angular rate during attitude acquisition.

the satellite to the desired attitude. In particular, note that the transient of the attitude quaternion (Fig. 5) shows that the transition from the initial to the final orientation of the satellite is carried out via an almost pure rotation around the $z$ body axis, i.e., the (initially correct) orientation of the $x$ and $y$ axis is only minimally perturbed. Finally, in Fig. 7 the behaviour of the elements of the (symmetric) matrix $\hat{\Gamma}_{\mathrm{av}}$ is shown. As can be seen from the figure, the elements of the estimated average gain converge to constant values for $t \rightarrow \infty$.

\section{CONCLUDING REMARKS}

An adaptive, state feedback proportional derivative-like control law for the magnetic attitude stabilisation of Earth-pointing spacecraft has been proposed. The novel control law has 

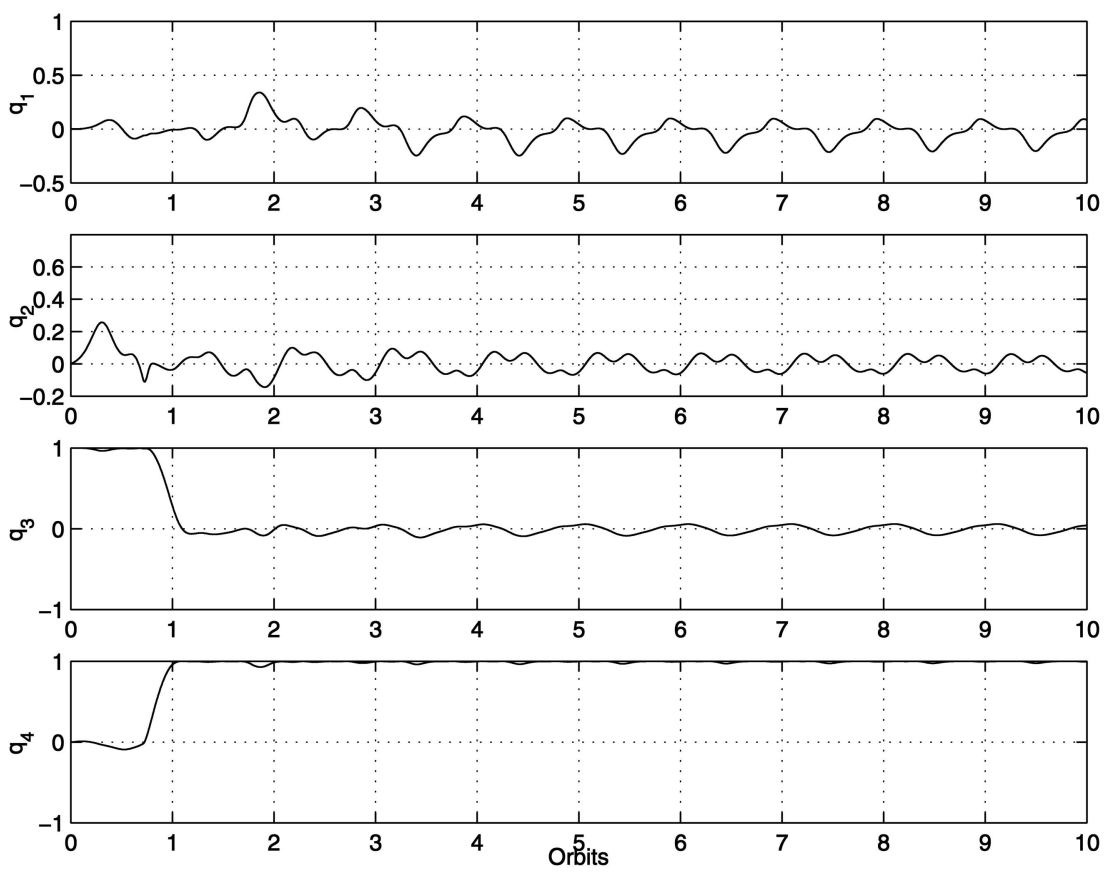

Fig. 5. Time evolution of quaternion during recovery from "upside down" attitude.
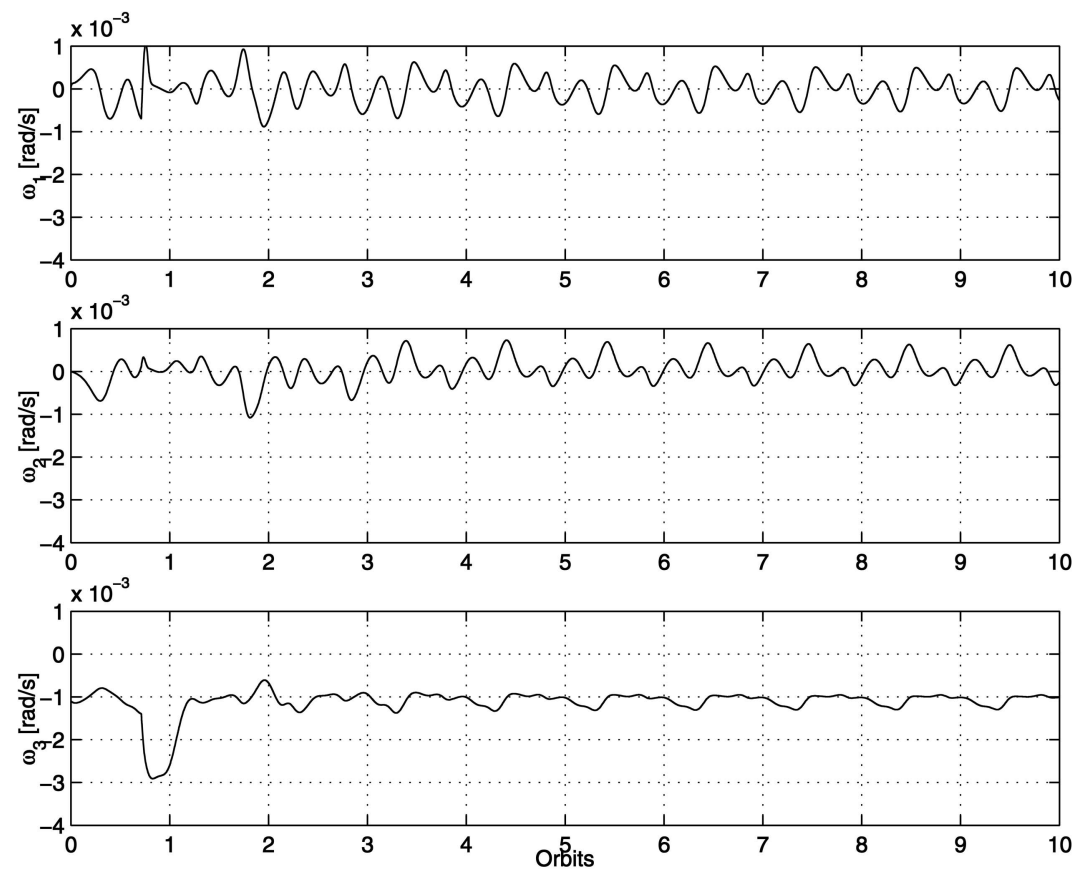

Fig. 6. Time evolution of absolute angular rate during recovery from "upside down" attitude.

been shown to guarantee almost global stability of the desired Earth-pointing equilibrium in the presence of gravity gradient torques acting on the satellite. Simulation results demonstrate the feasibility of the proposed approach and in particular illustrate the ability of the adaptive controller to bring the spacecraft to the desired relative Earthpointing attitude from an arbitrary initial condition.

\section{REFERENCES}

[1] White, J., Shigemoto, F., and Bourquin, K.

Satellite attitude control utilizing the Earth's magnetic field.

Technical Note D-1068, NASA, 1961.

[2] Stickler, A., and Alfriend, K.

An elementary magnetic attitude control system.

Journal of Spacecraft and Rockets, 13, 5 (1976), 282-287. 


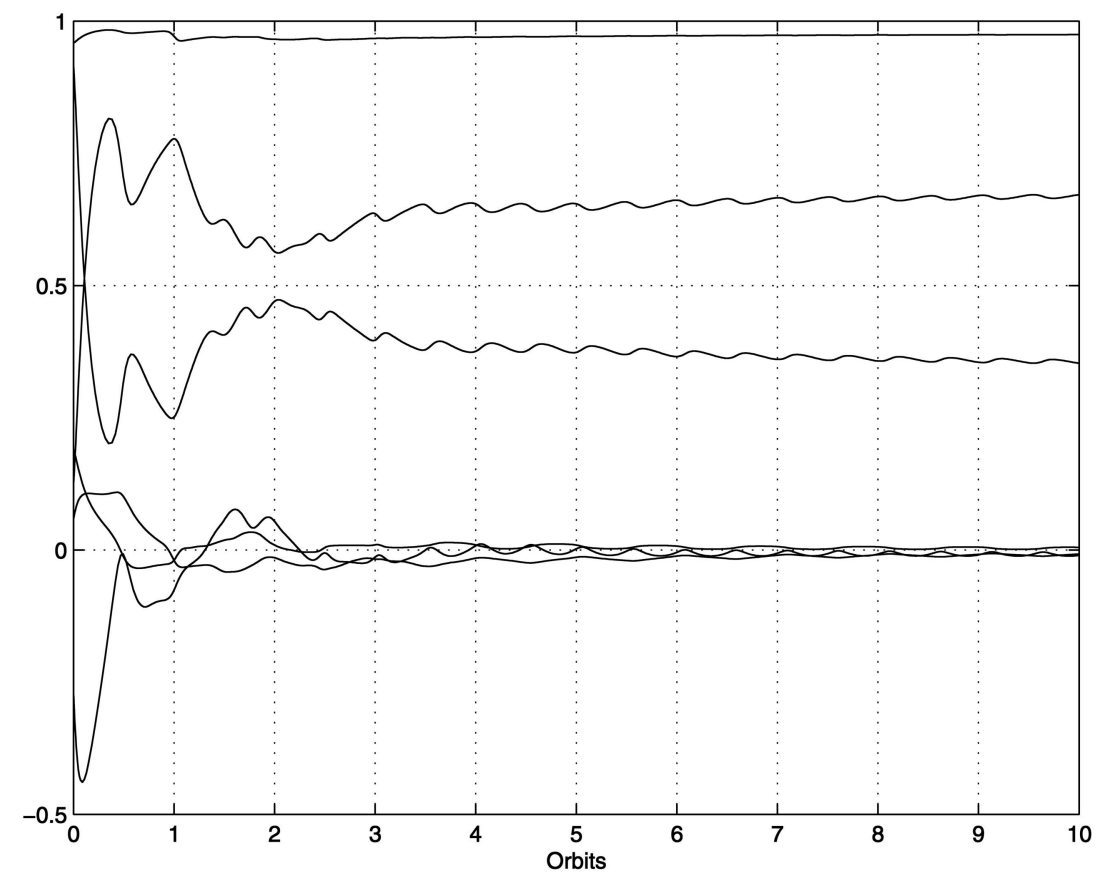

Fig. 7. Time evolution of elements of estimated average gain during recovery from "upside down" attitude.

[3] Pittelkau, M.

Optimal periodic control for spacecraft pointing and attitude determination.

Journal of Guidance, Control and Dynamics, 16, 6 (1993), 1078-1084.

[4] Wisniewski, R., and Markley, L.

Optimal magnetic attitude control.

Presented at the 14th IFAC World Congress, Beijing, China, 1999.

[5] Lovera, M., Marchi, E. D., and Bittanti, S.

Periodic attitude control techniques for small satellites with magnetic actuators.

IEEE Transactions on Control Systems Technology, 10, 1 (2002), 90-95.

[6] Psiaki, M.

Magnetic torquer attitude control via asymptotic periodic linear quadratic regulation.

Journal of Guidance, Control and Dynamics, 24, 2 (2001), 386-394.

[7] Steyn, W. H

Comparison of low Earth orbit satellite attitude controllers submitted to controllability constraints.

Journal of Guidance, Control and Dynamics, 17, 4 (1994), 795-804.

[8] Curti, F., and Diani, F.

Study on active magnetic attitude control for the Italian spacecraft bus MITA.

In Proceedings of the 14th International Conference on Spaceflight Dynamics, Iguassu Falls, Brazil, 1999.

[9] Wisniewski, R., and Blanke, M.

Fully magnetic attitude control for spacecraft subject to gravity gradient.

Automatica, 35, 7 (1999), 1201-1214.

[10] Damaren, C. J.

Comments on "Fully magnetic attitude control for spacecraft subject to gravity gradient."

Automatica, 38, 12 (2002), 2189.

[11] Arduini, C., and Baiocco, P.

Active magnetic damping attitude control for gravity gradient stabilised spacecraft.

Journal of Guidance and Control, 20, 1 (1997), 117-122.
[12] Wang, P., and Shtessel, Y.

Satellite attitude control using only magnetic torquers. Presented at the AIAA Guidance, Navigation, and Control Conference and Exhibit, Boston, 1998.

[13] Lovera, M., and Astolfi, A. Global attitude regulation using magnetic control. Presented at the IEEE Conference on Decision and Control, Tampa, Florida, 2001.

[14] Astolfi, A., and Lovera, M.

Global spacecraft attitude control using magnetic actuators.

Presented at the 2002 American Control Conference, Anchorage, Alaska, 2002.

[15] Lovera, M., and Astolfi, A. Spacecraft attitude control using magnetic actuators. Automatica, 40, 8 (2004).

[16] Wertz, J.

Spacecraft attitude determination and control. Dordrecht, The Netherlands, D. Reidel Publishing Company, 1978.

[17] Psiaki, M

Magnetic torquer attitude control via asymptotic periodic linear quadratic regulation.

Presented at the AIAA Guidance, Navigation, and Control Conference, Denver, Colorado, 2000.

[18] Khalil, H.

Nonlinear Systems (3rd ed).

Englewood Cliffs, NJ: Prentice Hall, 2001.

[19] Hughes, P.

Spacecraft Attitude Dynamics.

New York: Wiley, 1986.

[20] Wen, J. T-Y., and Kreutz-Delgado, K.

The attitude control problem.

IEEE Transactions on Automatic Control, 36, 10 (1991), $1148-1162$.

[21] Wisniewski, R.

Satellite attitude control using only electromagnetic actuation.

Ph.D. dissertation, Aalborg University, Denmark, 1996.

[22] Chobotov, V.

Spacecraft Attitude Dynamics and Control.

Orbit Books, 1991. 


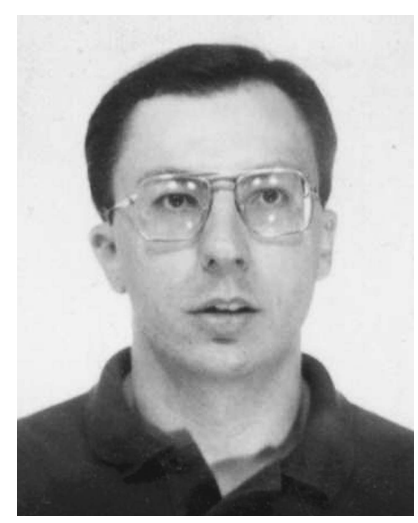

Marco Lovera was born in Milan, Italy, in 1967. He obtained the Laurea degree in 1993 and the Ph.D. in 1998, both in electronic engineering from the Politecnico di Milano, Italy.

He joined Carlo Gavazzi Space SpA, Milan, Italy, in May 1998, as control engineering specialist, working on spacecraft attitude determination and control systems and he contributed to the design of the attitude control software for the Italian spacecraft MITA and AGILE.

Since January 2004 he is associate professor at the Dipartimento di Elettronica e Informazione of the Politecnico di Milano. His main research interests include linear and nonlinear system identification, and advanced active control applications in aerospace, with specific reference to spacecraft attitude determination and control and active control of helicopter rotors.

Dr. Lovera is an associate editor of the IEEE Transactions on Control Systems Technology and a member of the IFAC Technical Committee on Control Design and of the IEEE CSS Technical Committee on Computer Aided Control Systems Design.

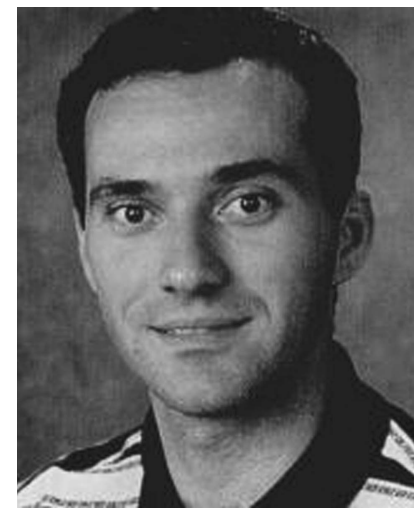

Alessandro Astolfi (M'98-SM'02) was born in Rome, Italy, in 1967. He graduated in electrical engineering from the University of Rome in 1991, and received the M.Sc. degree in information theory and the Ph.D. degree with Medal of Honor, both from ETH-Zurich, Switzerland, in 1992 and 1995, respectively. He also received the Ph.D. degree from the University of Rome for his work on nonlinear robust control in 1996.

Since 1996, he has been with the Electrical and Electronic Engineering Department of Imperial College, London, UK, where he is currently a professor in nonlinear control theory. From 1998 to 2003, he was also an associate professor with the Department of Electronics and Information of the Politecnico of Milan, Milan, Italy. Since 2005 he is also a full professor in the Department of Computer Science, Systems and Production of the University of Rome 'Tor Vergata.' He has been a visiting lecturer in nonlinear control at several universities, including ETH-Zurich $(1995,1996)$; Third University of Rome (1996); Rice University, Houston, TX (1999); Kepler University, Linz (2000); and SUPELEC, Paris (2001). His research interests are focused on mathematical control theory and control applications, with special emphasis on the problems of discontinuous stabilization, robust stabilization, robust control, and adaptive control.

Dr. Astolfi is the author of more than 50 journal papers, 15 book chapters, and over 100 papers in refereed conference proceedings. He is coeditor of a book on modeling and control of mechanical systems and editor of a book on nonlinear and adaptive control. He is also an Associate Editor of Systems and Control Letters, Automatica, the IEEE Transactions on Automatic Control, the International Journal of Control, and the European Journal of Control. He has also served in the IPC of various international conferences. 